\title{
Perceptions and Awareness of Overlapping Surgery in Patients With Shoulder Pain Presenting to an Orthopaedic Sports Medicine Clinic
}

\author{
Robert N. Matar, M.D., Brian Johnson, B.S., Nihar S. Shah, B.S., and Brian M. Grawe, M.D.
}

\begin{abstract}
Purpose: To determine patients' baseline understanding of overlapping surgery and to evaluate how education changes the perception of the practice in orthopaedic shoulder pain patients at a single institution. Methods: All patients who visit the clinic with a chief complaint of shoulder pain were given a 15-question survey. The initial 15-question survey assessed demographics, pre-existing knowledge on the practice of overlapping surgery, and their perception of it. They immediately read a statement on the practice of overlapping surgery. After reading the statement, patients were re-evaluated on their level of concern. Results: A total of 100 patients ( 55 female, 45 male) completed the survey. Mean age was 53.0 (range, $18-85)$ years. In total, 38 (38\%) had no knowledge on the practice of overlapping surgery; $27(27 \%)$ reported their level of concern as a 1, the lowest level of concern. Overall, $84(84 \%)$ patients reported a level of concern of 3 (median) or lower, indicating a low level of concern. A total of $95(95 \%)$ patients reported either a decrease or no change in level of concern after reading an educational statement on overlapping surgery practices, and $60(60 \%)$ believed there would be no impact if an overlapping surgery was performed. If a patient reported a high level of concern, the most common reasons cited were that the attending physician may not be available during the whole case (15\%); that a resident, fellow, or physician assistant may jeopardize the patient's care $(24 \%)$; or that a critical step would be missed $(37 \%)$. Conclusions: There is a low level of baseline understanding of overlapping surgery in patients with shoulder pain. An educational component added during patient counseling proved to be effective in decreasing the level of concern. This study suggests that counseling and education on overlapping surgery may change patient perception and opinion of the practice. Clinical Relevance: Serves as an evaluation of the knowledge of a specific patient population on overlapping surgery and how it changes with counseling and education.
\end{abstract}

$\mathbf{O}$ verlapping surgery is a common practice in highvolume settings such as academic medical centers. ${ }^{1}$ While this has been common practice for some time, public recognition has increased with landmark publications such as the 2015 Boston Globe article. The story described the process of overlapping surgery at the Massachusetts General Hospital and raised concerns

From the Department of Orthopaedics and Sports Medicine, University of Cincinnati, Cincinnati, Ohio, U.S.A.

The authors report that they have no conflicts of interest in the authorship and publication of this article. Full ICMJE author disclosure forms are available for this article online, as supplementary material.

Received September 9, 2019; accepted August 5, 2020.

Address correspondence to Robert N. Matar, M.D., Department of Orthopaedics and Sports Medicine, 231 Albert Sabin Way, Cincinnati, OH 452670212.E-mail:matarrt@ucmail.uc.edu

(C) 2020 THE AUTHORS. Published by Elsevier Inc. on behalf of the Arthroscopy Association of North America. This is an open access article under the CC BY-NC-ND license (http://creativecommons.org/licenses/by-nc-nd/4.0/). 2666-061X/191100

https://doi.org/10.1016/j.asmr.2020.08.001 over patient safety and outcomes. ${ }^{2}$ Another report by the Seattle Times on the practice in Sweden also questioned whether patient safety was endangered and if financial gain played a role. ${ }^{3}$

While the terms "overlapping" and "concurrent" surgery are similar, they are different in terms of legality. Concurrent surgery is when a surgeon is responsible for critical or key parts of more than one surgery occurring at the same time. In contrast, it is considered overlapping surgery when only noncritical parts of more than one surgery are occurring at the same time. ${ }^{4}$ As it stands today, the Centers for Medicare and Medicaid Services billing does not condone concurrent surgery.

The medical community believes overlapping surgery is important for resident education in addition to being more economically sound for the hospital in terms of time and costs. ${ }^{6,7}$ Simultaneously, legislators have sought to understand the practice and identify institutional policies at hospitals across the country. In 2016 the Senate Financial Committee identified a large 
Table 1. Demographic Data for the Population of Study

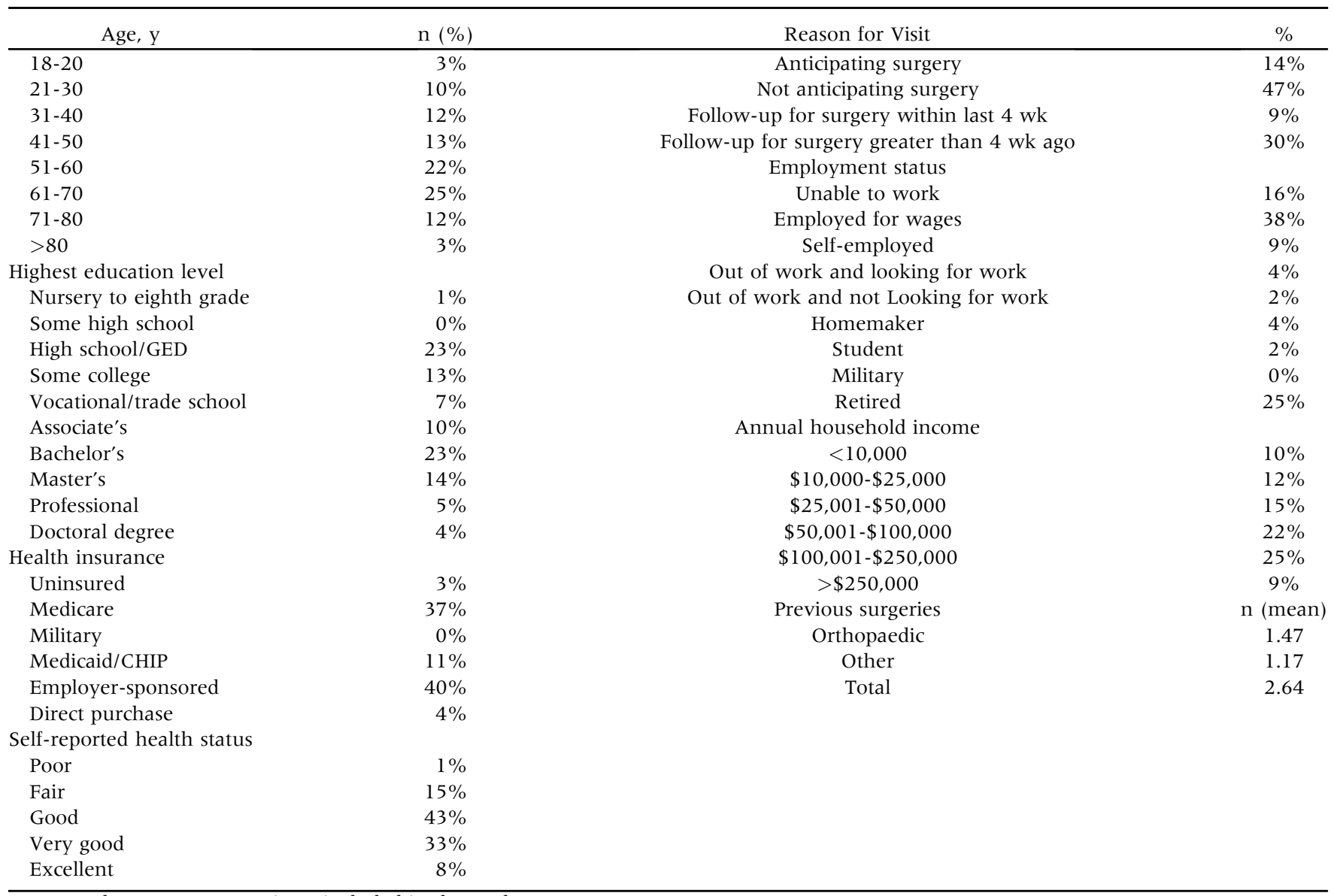

NOTE. There were 100 patients included in the study.

CHIP, Children's Health Insurance Program; GED, General Educational Development.

percentage of hospitals did not have policies in place. The investigation found inadequacies in defining the practice to patients and developing a protocol to ensure back up surgeons are available. ${ }^{5}$ Similarly, a survey of 1400 patients showed that $4 \%$ knew what overlapping surgery was yet $95 \%$ believed that it should be disclosed preoperatively. ${ }^{8}$

Since the Senate's report, the American College of Surgeons has recommended that providers disclose the use of overlapping surgeries to all patients, regardless of which part of the surgery is overlapped. Despite the significance this topic has on patients, physicians, legislators, and insurance companies alike, very few studies have measured the general awareness of the practice with patients and the changes in perception after education. The purpose of this study was to determine patients' baseline understanding of overlapping surgery and to evaluate how education changes the perception of the practice in orthopaedic shoulder pain patients at a single institution. We hypothesized that most patients would have no baseline understanding of overlapping surgery practices and that patient education would cause a reduction in concern.

\section{Methods}

Institutional review board approval was gained for both the study design and the survey that was used in this study (University of Cincinnati, manuscript number: 2019-0665). Inclusion criteria included a patient presenting with a chief complaint of shoulder pain. Patients who did not use English as their first language were excluded, but there was no exclusion made on the basis of age, history of previous surgery, or insurance status. All eligible patients were seen in a single orthopaedic sports medicine clinical practice over the course of 3 months (June 2019-August 2019). Patients at this office were not informed of the practice of overlapping surgery before the encounter. The nondemographic survey questions and answers were assessed to be at an eighth-grade reading level according to the Flesch-Kinkaid readability test. Before seeing the physician, one author (B.J.) approached the first 100 eligible patients and asked if they would be interested in completing a survey about their knowledge of the practice of overlapping surgery. The survey (Appendix) contained demographic questions as well as 6 questions to assess perceptions of overlapping 


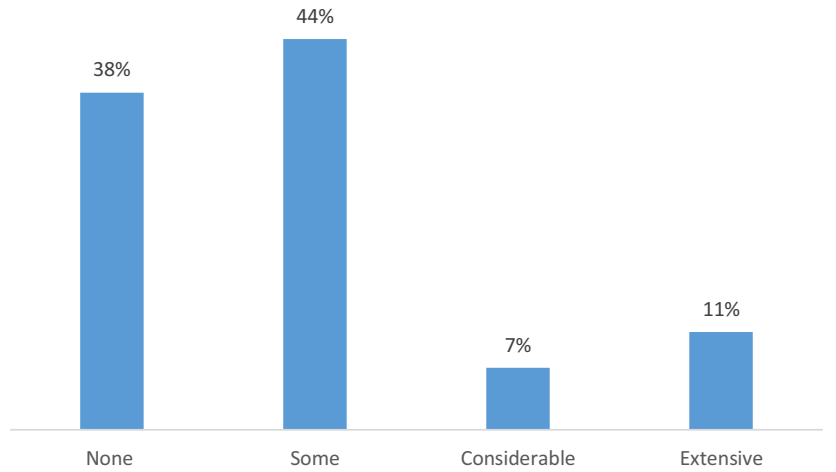

Fig 1. Patients were given 4 options when asked about their knoweldge of the practice of overlapping surgery: none, some, considerable, and extensive. There were 100 responses.

surgery. The 6 questions were taken from Evans et al. ${ }^{9}$ The author (B.J.) then provided a written educational statement that was taken from the study of Evans et al. that is shown to follow:

"At times our surgeons will work back and forth between two operating rooms in a staggered order. This means that as one surgery is finishing with the closure, putting on the dressing, waking up from anesthesia, and going to recovery, the surgeon can start working on a patient in another room. Your surgeon will always be present for all of the critical parts of the surgery. Our physician assistants, residents, and fellows are involved with assisting in surgery and getting patients ready in the operating room, but your faculty surgeon is your primary surgeon on every case. This staggered arrangement enhances efficiency and reduces wait times for scheduling your surgery. If you have questions or concerns about this process, your surgeon is happy to discuss them with you."

It was ensured that the patient understood the information that was being given, and any questions were answered with effort to not provide any more information that was included in the statement. After reading the statement, patients were then asked to rerate their level of concern and their changes in perception on the practice.

\section{Statistical Analysis}

Descriptive statistics such as mean and range calculations were used on continuous variables. Continuous variables were compared with each other using $t$ tests. Frequencies and percentages were calculated for categorical variables. $\chi^{2}$ tests were used to compare categorical variables such as education level, previous surgical history, and questions relating to perceptions of overlapping surgery. Significance was set to $P<.05$.

\section{Results}

There were 100 patients included in the study. The mean patient age was 53.0 (range, 18-85) years. In total, $45 \%(\mathrm{n}=45)$ were male patients and $55 \%$ $(\mathrm{n}=55)$ were female (Table 1$)$. Study subjects were most commonly the nonoperative patient not anticipating surgery $(47 \%)$. The remaining were either anticipating surgery (14\%), were scheduled for a follow-up surgery within the last 4 weeks $(9 \%)$, or a postoperative follow-up greater than 4 weeks (30\%). Insurance and employment status are denoted in Table 1 . In total, $58 \%(n=58)$ had never visited an orthopaedic surgeon before. In total, $45 \%(n=45)$ had not done previous research online or in-print before being seen in the clinic and $47 \%(n=47)$ had not prepared questions beforehand. A total of $84 \%(n=84)$ rated their general health as good or better.

A total of $38(38 \%)$ had no knowledge on the practice of overlapping surgery (Fig 1), 44 (44\%) reported some knowledge on the practice, and only $11(11 \%)$ had extensive understanding. In total, 62 patients $(62 \%)$ reported at least some baseline understanding and awareness on the practice of overlapping surgery.

In total, $27(27 \%)$ reported their level of concern as a 1 , corresponding to the lowest level of concern. Overall, 84 of the $100(84 \%)$ patients reported a level of concern of 3 (median) or lower, indicating a low mean level of concern (Fig 2).

After reading an educational statement on overlapping surgery practices, $95(95 \%)$ patients reported either a decrease or no change in level of concern (Fig 3). More specifically, 43 patients (43\%) reported no change in their level of concern, whereas $52(52 \%)$ reported a decrease. Five $(5 \%)$ reported an increase in their level of concern.

Sixty $(60 \%)$ believed there would be no impact if overlapping surgery was performed (Fig 4). If a patient reported concern, the most common reasons cited were that the attending physician may not be available during the whole case $(15 \%)$, that a resident, fellow, or physician assistant may jeopardize the patient's care $(24 \%)$, or that a critical step will be done incorrectly (37\%) (Fig 5).

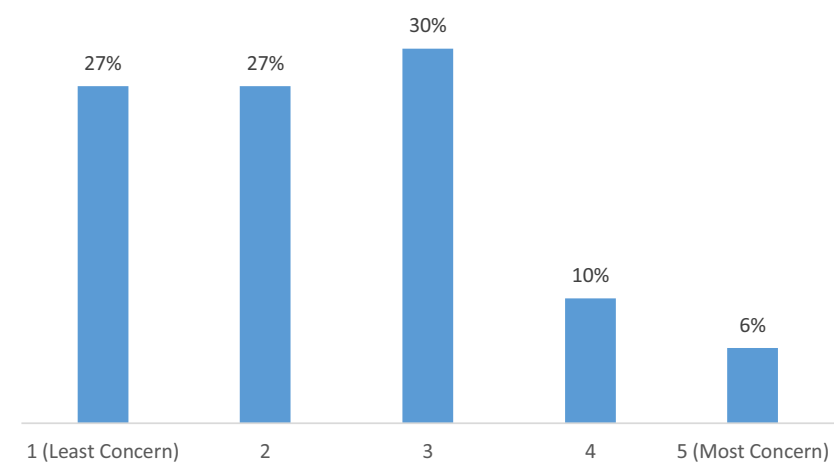

Fig 2. Patients rated the level of conern from 1 to 5 when asked how the felt about receiving surgery that overloaps with someone elses. There were 100 responses. 


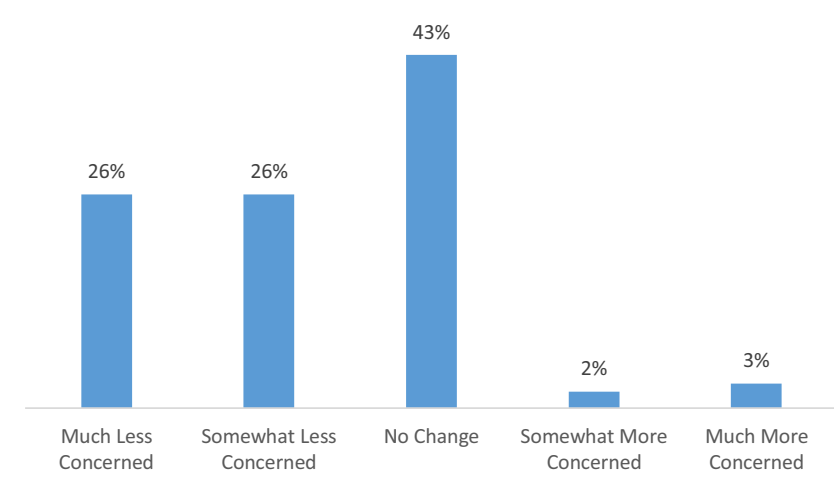

Fig 3. Patients rated the change in their level of conern after they were given an educational statement about the practice of overlapping surgery. There were 5 options: much less concerned, somewhat less concerned, no change, somewhat more concerned, and much more conerned. There were 100 responses.

We also aimed to test the relationship between certain demographic features and data on baseline understanding and concern, and the change in concern on overlapping surgery. Some of those demographic features include previous surgical history, education level, research before clinical encounter, and questions prepared before the clinical encounter.

Patients who had a previous surgical history were associated with a greater baseline understanding $(P<.001)$ and lower level of concern $(P<.001)$. Patients performing research on their condition before visiting the physician were not associated with an improved baseline understanding and lower level of concern $(P=.11$ and .40 , respectively). Research about the patient's chief complaint before the clinical encounter was not associated with an improved baseline understanding and lowered concern $(P=.05$ and .10 , respectively). Those who had someone accompany them were not associated with an improved baseline understanding $(P=.59)$; however, there was a significant relationship with those same patients and a reduced baseline concern $(P<.05)$. Lastly, there was no association between education level and a more pronounced reduction in concern after reading an educational statement $(P=.93)$.

\section{Discussion}

Awareness on the practice of overlapping surgery and its influence on resident education, patient care, and economic efficiency is increasing, as it affects many populations. In this study, we evaluated the baseline knowledge on overlapping surgery and the affect patient education has on changes in perception and concern. In addition, we aimed to identify any demographic features and risk factors that could be associated and influence baseline patient understanding and level of concern as well as serve as a predictor for postoperative concern and outcomes.
Our results demonstrated that $95 \%$ of patients had a reduction in their level of concern after counseling and education. Sixty percent of patients believed there was no impact of overlapping surgery and $26 \%$ believed it would have a positive impact on their outcome. History of previous surgery was associated with a self-perceived better understanding of the concept of overlapping surgery $(P<.001)$ and a lower level of concern with the practice $(P<.001)$. Patients who were accompanied to their office visit rated themselves as having a lower level of concern $(P<.05)$.

The data also indicated that a previous surgical history may inform patients of the practice of overlapping surgery and in conjunction reduce concern the next time they may need surgery. In addition, encouraging family and friends to accompany patient's during preoperative counseling may help reduce concern on the practice. Research before the clinical encounter was not associated with a greater baseline understanding and lower level of concern. We had previously thought that patient research and preparation may populate results on the topic and increase patient perception of awareness.

Previous literature has evaluated overlapping surgery; however, the focus has been primarily on the relationship between overlapping surgery and patient outcomes. These studies were performed in a number of specialties, such as neurosurgery, ${ }^{10-14}$ head and neck surgery, ${ }^{15,16}$ orthopaedic surgery, ${ }^{17-19}$ pediatric surgery, ${ }^{20}$ and general surgery. ${ }^{9,21-23}$ However, these studies did not address patient perception of what overlapping surgery is and how it affects the studied outcome.

\section{Limitations}

The limitations of this study are inherent in its design. Although this study took place at 3 different clinics to capture a full spectrum of patient demographics, it was performed at the offices of 1 orthopaedic subspecialist. We imagine the policies and preoperative counseling can be substantially different among institutions and

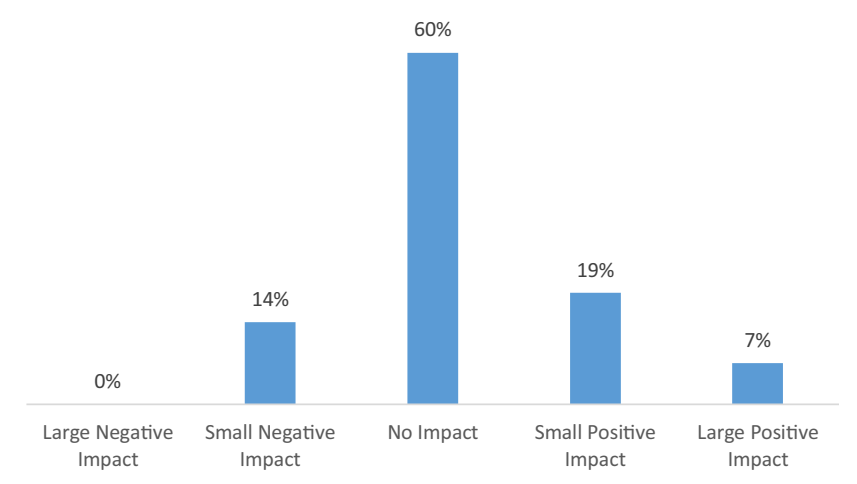

Fig 4. Patients rated how they thought overlapping surgery would impact their care if they were to have surgery. They were given 5 choices: large negative impact, small negative impact, no impact, small positive impact, and large positive impact. There were 100 responses. 


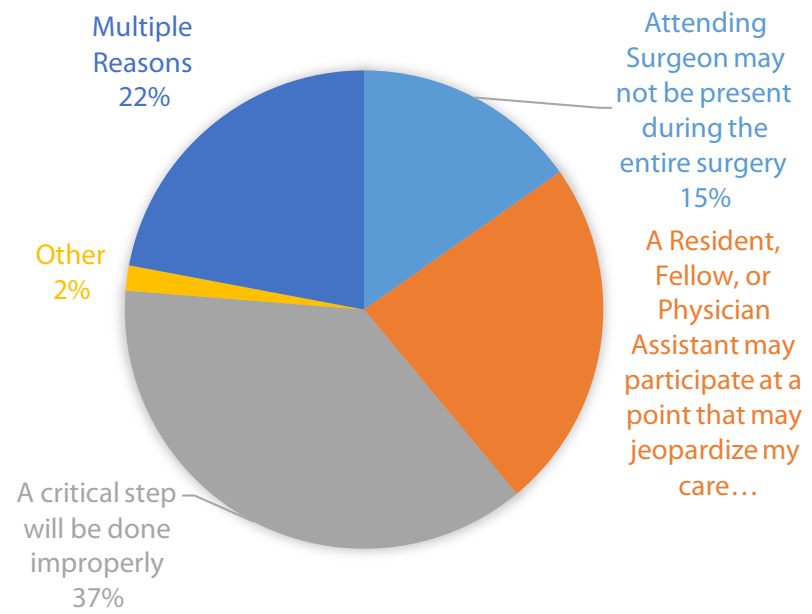

Fig 5. Patients were asked what their concern would be if they were to have an overlapping surgery. There were 4 choices: the attending surgeon may not be present during the entire surgery; a resident, fellow, or physician assistant may participate at a point that may jeopardize my care; a critical step will be done improperly; or other. They were permitted to select multiple choices. There were 100 responses.

physicians. In addition, there was no way of controlling level of patient education of overlapping surgery before the survey in the study design. Some of the patients included in our analysis were there for their initial postoperative visit and were likely patients of overlapping surgery. These patients may have had more confidence in their knowledge of overlapping surgery due to informed consent before the surgery. Finally, we did not have a control group of patients with whom to compare our results.

The reading level of our survey was assessed to be of grade level 8. Although this was not ideal, we attempted to make our survey as clear and concise as possible without sacrificing our ability to assess patient opinion. In addition, while one author always accompanied the patient when they filled out the questionnaire and read the educational statement, we did not ensure that patients had adequate literacy before we administered the survey.

\section{Conclusions}

There is a low level of baseline understanding of overlapping surgery in patients with shoulder pain. An educational component added during patient counseling proved to be effective in decreasing the level of concern. This study suggests that counseling and education on overlapping surgery may change patient perception and opinion of the practice.

\section{References}

1. Theriault B, Pazniokas J, Mittal A, et al. What does it mean for a surgeon to "run two rooms?" A comprehensive literature review of overlapping and concurrent surgery policies. Am Surg 2019;85:420-430.
2. Abelson J, Saltzmann J, Kowalcyzk L, Allen S. Clash in the name of care-A Boston Globe Spotlight Team Report, https://apps.bostonglobe.com/spotlight/clash-inthe-name-of-care/story/. Accessed July 31, 2019. Published 2015.

3. Baker M. Swedish double-booked its surgeries, and the patients didn't know The Seattle Times. The Seattle Times, https://www.seattletimes.com/seattle-news/times-watch dog/swedish-neuroscience-institute-double-booked-over lapping-surgeries/. Accessed August 13, 2019. Published 2017.

4. Statements on Principles. Bulletin of the American College of Surgeons, https://www.facs.org/about-acs/statements/ stonprin. Accessed July 31, 2019. Published 2016.

5. Senate Finance Committee Staff. Concurrent and Overlapping Surgeries: Additional Measures Warranted A Senate Finance Committee Staff Report. 2016, https:// Www.finance.senate.gov/download/finance-concurrentsurgeries-report. Accessed August 13, 2019.

6. Langerman A. Concurrent surgery and informed consent. JAMA Surg 2016;151:601.

7. Beasley GM, Pappas TN, Kirk AD. Procedure delegation by attending surgeons performing concurrent operations in academic medical centers: Balancing safety and efficiency. Ann Surg 2015;261:1044-1045.

8. Kent M, Whyte R, Fleishman A, Tomich D, Forrow L, Rodrigue J. Public perceptions of overlapping surgery. J Am Coll Surg 2017;224:771-778.e4.

9. Evans CL, Kahler DM, Weiss DB, Yarboro SR. Understanding and perception of overlapping surgery in an orthopaedic trauma population. J Am Acad Orthop Surg 2019;27:e473-e481.

10. Bohl MA, Mooney MA, Sheehy JP, et al. Overlapping surgeries are not associated with worse patient outcomes: Retrospective multivariate analysis of 14872 neurosurgical cases performed at a single institution. Neurosurgery 2018;83:53-59.

11. Guan J, Brock AA, Karsy M, et al. Managing overlapping surgery: An analysis of 1018 neurosurgical and spine cases. J Neurosurg 2017;127:1096-1104.

12. Howard BM, Holland CM, Mehta CC, et al. Association of overlapping surgery with patient outcomes in a large series of neurosurgical cases. JAMA Surg 2018;153:313.

13. Mooney MA, Brigeman S, Bohl MA, et al. Analysis of overlapping surgery in patients undergoing microsurgical aneurysm clipping: Acute and long-term outcomes from the Barrow Ruptured Aneurysm Trial. I Neurosurg 2018;129:711-717.

14. Zygourakis CC, Lee J, Barba J, Lobo E, Lawton MT. Performing concurrent operations in academic vascular neurosurgery does not affect patient outcomes. J Neurosurg 2017;127:1089-1095.

15. Zygourakis CC, Keefe M, Lee J, et al. Comparison of patient outcomes in 3725 overlapping vs 3633 nonoverlapping neurosurgical procedures using a single institution's clinical and administrative database. Neurosurgery 2017;80:257-268.

16. Sweeny L, Rosenthal EL, Light T, et al. Effect of overlapping operations on outcomes in microvascular reconstructions of the head and neck. Otolaryngol Neck Surg 2017; 156:627-635. 
17. Hamilton WG, Ho H, Parks NL, et al. The Lawrence D. Dorr Surgical Techniques \& Technologies Award: "Running two rooms" does not compromise outcomes or patient safety in joint arthroplasty. J Arthroplasty 2018;33:S8-S12.

18. Ravi B, Pincus D, Wasserstein D, et al. Association of overlapping surgery with increased risk for complications following hip surgery. JAMA Intern Med 2018;178:75.

19. Zhang AL, Sing DC, Dang DY, et al. Overlapping surgery in the ambulatory orthopaedic setting. J Bone Joint Surg 2016;98:1859-1867.
20. Hyder JA, Hanson KT, Storlie CB, et al. Assessing the safety of overlapping surgery at a children's hospital. Ann Surg 2018;268:e24-e27.

21. Hyder JA, Hanson KT, Storlie CB, et al. Safety of overlapping surgery at a high-volume referral center. Ann Surg 2017;265:639-644.

22. Liu JB, Berian JR, Ban KA, et al. Outcomes of concurrent operations. Ann Surg 2017;266:411-420.

23. Ponce BA, Wills BW, Hudson PW, et al. Outcomes with overlapping surgery at a large academic medical center. Ann Surg 2019;269:465-470. 